\title{
Insuficiencia cardiaca con fracción de eyección conservada: ¿Cómo podemos abordarla?
}

\section{Heart failure with preserved ejection fraction: how can we approach it?}

Juan David Orozco-Burbano ${ }^{1}$, Carlos Ernesto Cabrera-Velasco ${ }^{1}$, Juan Isaac Ortiz-Andrade ${ }^{2}$, Nelson Adolfo López-Garzón ${ }^{3}$

\section{RESUMEN}

La insuficiencia cardiaca con fracción de eyección preservada constituye un síndrome clínico con una prevalencia estimada entre el $1 \%$ al $5.5 \%$, el cual se asocia con edad avanzada, diversos mecanismos inflamatorios $y$ alteraciones metabólicas, que propician cambios

\begin{abstract}
Heart failure with preserved ejection fraction is a clinical syndrome with an estimated prevalence between $1 \%$ and $5.5 \%$, which is associated with advanced age, multiple inflammatory mechanisms, and metabolic alterations thatpromote structural typical alterations of this condition.
\end{abstract}

\section{Historial del artículo:}

Fecha de recepción: 26/01/2020

Fecha de aprobación: 10/06/2020

1 Universidad del Cauca, Facultad de Ciencias de la Salud. Residente de Medicina Interna, Popayán, Colombia.

2 Universidad del Cauca, Facultad de Ciencias de la Salud. Internista. Profesor del Departamento de Medicina Interna, Popayán, Colombia.

3 Universidad del Cauca, Facultad de Ciencias de la Salud. Internista, Cardiólogo, Ecocardiografista. Profesor del Departamento de Medicina Interna, Popayán, Colombia.

Correspondencia: Juan David Orozco Correo electrónico: jdorozco@unicauca.edu.co. Departamento de Medicina Interna, Universidad del Cauca. Popayán, Colombia.

Como citar este artículo: Orozco-Burbano JD, Cabrera-Velasco CE, Ortiz-Andrade J, López-Garzón N. Insuficiencia cardiaca con fracción de eyección conservada: ¿Cómo podemos abordarla? Revista de la Facultad de Ciencias de la Salud de la Universidad del Cauca. 2020;22(2):26-33. https://doi.org/10.47373/rfcs.2020.v22.1489 
estructuralespropios de esta condición. Se ha establecido para su diagnóstico una fracción de eyección mayor al $50 \%$ y marcadores de disfunción diastólica valorados por ecocardiografía. El abordaje de este síndrome es complejo dada la variabilidad de fenotipos que pueden presentarse clínicamente $y$, por esto, se han diseńado diversas estrategias para favorecer un adecuado diagnóstico y tratamiento, como escalas predictivas, métodos imagenológicos no invasivos ybiomarcadores, entre otros. En este artículo de revisión narrativa, se realizó una búsqueda ampliada de la literatura con el fin de ofrecer un abordaje práctico de esta condición.

Palabras clave: Insuficiencia Cardíaca, Fracción de eyección, Biomarcadores, Fenotipo, Tratamiento. (DeCS)

\section{INTRODUCCIÓN}

La insuficiencia cardiaca con fracción de eyección preservada (ICFEp) constituye un síndrome clínico caracterizado por mantener una fracción de eyección del ventrículo izquierdo (FEVI) estimada por ecocardiografia transtorácica (EcoTT) igual o mayor al 50\% (1). Esta entidad puede representar hasta la mitad de pacientes que se presentan con insuficiencia cardiaca (IC). Actualmente, es controversial si ICFEp constituye un síndrome único o un conjunto de síndromes (2), puesto que se ha asociado con alteraciones fisiopatológicas, factores de riesgo y una presentación clínica que puede ser diferente a la de los pacientes con una fracción de eyección reducida (ICFEr). Además, cuenta con opciones farmacológicas limitadas, dado que las intervenciones existentes no han logrado demostrar una importante reducción de la morbilidad y la mortalidad asociada a esta patología (3-4). El propósito del artículo fue llevar a cabo una revisión narrativa de las características más relevantes de este síndrome, con el fin de lograr una aproximación diagnóstica y terapéutica con la evidencia disponible al momento, facilitando el abordaje de estos pacientes en la práctica clínica.

\section{MÉTODOS}

Las bases de datos que se revisaron fueron PubMed, Google Scholar y SciELO, estableciendo un límite de tiempo de 10 ańos, sin restricción idiomática (desde el 01/01/2010, hasta el 01/10/2020). Las palabras clave utilizadas fueron: Heart
The diagnosis of this heart failure has been established as an ejection fraction $>50 \%$ and diastolic dysfunction markers through the echocardiography. The approach to this syndrome is complex due to the variability of phenotypes that can appear clinically. A lot of strategies have been designed to favor an adequate diagnosis and treatment, such as predictive scales, non-invasive imaging methods, biomarkers, among others. In this narrative review article, we performed an expanded search of the literature to give apractical approach to this condition.

Keywords: Heart failure, Ejection fraction, Biomarkers, Phenotype, Treatment. (MeSH)

failure, Preserved ejection function, Biomarkers, Phenotype and treatment, las cuales fueron combinadas con el término booleano "AND". La búsqueda arrojó un total de 54 artículos, los cuales incluían en su título principal o en su resumen las palabras clave. Se excluyeron los artículos que no tuvieran disponible el texto completo o no tuvieran relación con el objetivo de esta revisión, estudios duplicados, únicamente descriptivos y reportes de casos.

\section{EPIDEMIOLOGÍA}

Se ha estimado una prevalencia entre $1.1 \%$ y $5.5 \%$ de ICFEp en la población general, con un constante incremento de $1 \%$ por ańo (5), así como una incidencia de 650.000 nuevos casos por ańo (6). Sin embargo, la estimación precisa de la frecuencia de este síndrome ha sido un reto debido a la falta de estandarización de criterios diagnósticos, poblaciones estudiadas y a los diversos factores de riesgo asociados (7). Para Colombia, la ICFEp representa el $67.5 \%$ de los casos atendidos ambulatoriamente por IC (8). Es importante destacar que, asociado a descompensaciones que impactan en calidad de vida (10), estos pacientes tienen altas tasas de hospitalización, similares a pacientes con ICFEr (9). También presentan tasas similares de mortalidad que los pacientes con ICFEr; sin embargo, esta situación no es un hallazgo consistente (11). Las causas no cardiovasculares son la mayor causa de muerte en esta población (12). Respecto a las causas de mortalidad cardiovascular, el accidente cerebrovascular (8 a 14\%) y el infarto agudo de miocardio (5\%) son las más frecuentes (13). 


\section{FACTORES DE RIESGO}

La ICFEp es más prevalente en mujeres, con una distribución que puede ir hasta un 63\% de los casos (14). Entre los 25 y 49 ańos de edad, la prevalencia es del $1 \%$ en mujeres y del $0 \%$ en hombres y se eleva a $8-10 \%$ en mujeres y $4-6 \%$ en hombres de 80 ańos o más (5). Por otra parte, se ha asociado la presentación de ICFEp con importantes factores de riesgo, entre ellos hipertensión arterial, diabetes mellitus, enfermedad renal crónica, obesidad, miocardiopatía dilatada, fibrilación auricular y enfermedad coronaria $(8,15)$. Sobresalen los individuos que padecen hipertensión pulmonar y/o fibrilación auricular por su mayor intolerancia al ejercicio, hospitalizaciones frecuentes y menor supervivencia. Lo anterior obedece a que el incremento de $10 \mathrm{mmHg}$ en la presión arterial pulmonar se asocia con un aumento del $28 \%$ en la mortalidad a tres ańos y a que el llenado ventricular depende en gran medida de la contracción auricular en la ICFEp (16-18). La obesidad también se ha descrito como factor de riesgo relevante en estos pacientes, debido a un incremento en el remodelamiento cardiaco y disfunción diastólica (19).

\section{FISIOPATOLOGÍA}

La ICFEp está enmarcada por múltiples mecanismos desencadenantes y amplificación de dańo que resultan en un conjunto de signos y síntomas derivados de anomalías en la estructura y función cardiovascular $(13,20)$. La perturbación hemodinámica central es la disfunción diastólica, generada por una alteración en la relajación y aumento de la rigidez miocárdica, que conllevan a un aumento en las presiones de llenado ventricular izquierdo y posterior disfunción miocárdica $(1,21)$. Esta condición genera una disminución de la presión de perfusión coronaria, ocasionando isquemia subendocárdica que exacerba la disfunción (22). Sin embargo, la función sistólica del VI no es necesariamente normal. Existen alteraciones en la función y capacidad cardiaca para aumentar el volumen sistólico, asociado a una disminución en la distensibilidad arterial, que se presentan en reposo y empeoran con el ejercicio $(11,23)$.

Se han establecido alteraciones morfológicas y funcionales como la hipertrofia y rigidez del cardiomiocito junto con una fibrosis intersticial (20), derivadas de modificaciones a nivel bioquímico como el incremento en el colágeno fibrilar, alteraciones en la fosforilación de la titina, que conllevan a un inadecuado retroceso diastólico. Existe además una importante disfunción microvascular coronaria que afecta a los cardiomiocitos, disminuyendo la biodisponibilidad del óxido nítrico y mono fosfato de guanosina cíclica (GMPc) $(22,24)$. No obstante, la ICFEp no solo se debe asociar a un deterioro en la función del miocardio, sino a un conjunto de alteraciones cardiovasculares (endoteliales-ventriculares y vasculares) que conllevan a una disminución de la reserva cardiovascular total $(11,25)$.

La adiposidad juega un papel importante en la fisiopatología de esta condición. Por un lado, existe un incremento en las presiones de llenado ventricular por el aumento de masa corporal, junto con una disfunción endotelial derivada de una disminución en la producción de óxido nítrico, asociada a una inflamación sistémica de bajo grado $(11,26)$. Por otra parte, existe una disminución de la reserva ventilatoria pulmonar por un aumento en el espacio muerto junto a una reserva vasodilatadora pulmonar deteriorada, que se ha relacionado con una disminución en la capacidad del ejercicio (21), uno de los síntomas cardinales de los pacientes con ICFEp.

\section{DIAGNÓSTICO}

El diagnóstico de ICFEp se debe guiar inicialmente por los síntomas clínicos y una posterior evaluación ecocardiográfica $(27,28)$. El síntoma cardinal es la disnea durante el ejercicio, por lo cual el diagnóstico clínico es un reto y puede generar falsos positivos por otras causas comunes de disnea ante el ejercicio. Además, estos pacientes pueden presentarse clásicamente sin ortopnea o disnea nocturna paroxística o sin congestión manifiesta al examen clínico $(29,30)$.

La ecocardiografía permite la identificación de una FEVI mayor al 50\%, junto con el criterio adicional de la presencia de disfunción diastólica (DD) (3). Los hallazgos más comunes de este examen son: aumento en el volumen auricular izquierdo, evidencia de presión de llenado del ventrículo izquierdo elevada (relación E/e' elevada) y signos indirectos de hipertensión pulmonar (4,31). En individuos sin diagnóstico claro de ICFEp y con disnea inexplicable o intolerancia al ejercicio está indicado el ecocardiograma de estrés (1) y, en ocasiones, se pueden requerir estudios invasivos como el cateterismo cardiaco, el cual es el estándar de oro para el diagnóstico de DD y, además, ayuda a exclusión de otras causas de disnea no explicada $(1,32)$.

Considerando las dificultades en el diagnóstico, los biomarcadores surgen como herramientas de apoyo, siendo los más frecuentemente utilizados el péptido natriurético tipo $\mathrm{B}$ (BNP) y el pro-péptido natriurético tipo B (NT-proBNP), con un punto de corte de $100 \mathrm{pg} / \mathrm{mL}$ y $300 \mathrm{pg} / \mathrm{mL}$ respectivamente $(33,34)$. Sin embargo, estos pueden estar en rangos normales 
durante los periodos sin síntomas o bajos en comparación de pacientes con ICFEr por lo cual sus valores negativos no descartan el diagnóstico $(25,35,36)$. Cabe destacar que estos pueden estar alterados por diversas patologías, encontrando bajos niveles en pacientes obesos (37) o elevados en enfermedad renal crónica, fibrilación auricular, edad avanzada e incluso en mujeres sin otras comorbilidades, lo cual dificulta aún más su interpretación (33).

Actualmente se han descrito herramientas predictivas como la $\mathrm{H}_{2} \mathrm{FPEF}$, la cual estima la probabilidad de ICFEp mediante características clínicas simples y ecocardiografícas en pacientes con disnea de esfuerzo inexplicable, guiando al clínico a determinar la necesidad de más pruebas diagnósticas (cuando la puntuación de la escala da una probabilidad baja para ICFEp) (38). Al mismo tiempo, esta escala podría ser potencialmente útil en pacientes con puntuación alta, dado que podría predecir un alto riesgo de eventos cardiovasculares (39). Por otro lado, se encuentra la escala HFA-PEFF, la cual puede ayudar al diagnóstico de ICFEp, usando una serie de parámetros clínicos, funcionales, morfológicos y de laboratorio (biomarcadores). Se considera que una puntuación total de $\geq 5$ puntos es diagnóstica de ICFEp, mientras que una puntuación de $\leq 1$ punto hace que el diagnóstico sea muy improbable y se deben buscar otras etiologías. Los pacientes con una puntuación intermedia (2-4 puntos) necesitan una evaluación adicional con estudios invasivos para esclarecer el diagnóstico o descartar otras alteraciones $(40,41)$.

\section{TRATAMIENTO}

El manejo para esta condición aún no está claro, dado que la mayoría de fármacos que han sido usados en ICFEr no han mostrado una disminución de desenlaces cardiovasculares primarios ni en tasas de hospitalización, posiblemente por las diferencias en los mecanismos fisiopatológicos descritos (42). Por lo tanto, actualmente el tratamiento se enfoca al control de las comorbilidades con medidas no farmacológicas y farmacológicas (43).

Entre las medidas no farmacológicas, se destaca la actividad física con un tiempo y un consumo metabólico semanal mayor o igual a 150 minutos y de $350 \mathrm{MET} / \mathrm{min}$ semanal (siglas en inglés metabolic equivalent of task), permitiendo un aumento en la tolerancia al ejercicio, disminución en el riesgo de hospitalización por IC y mejoría en la calidad de vida $(44,45)$. Por otra parte, la disminución de peso por restricción calórica o ejercicio se asocia a un aumento en el consumo máximo de oxígeno con mejoría de los síntomas, sobretodo la disnea $(46,47)$.
En cuanto a los fármacos disponibles, en primer lugar, se deben considerar los medicamentos óptimos y necesarios para controlar las comorbilidades asociadas a ICFEp como hipertensión arterial, enfermedad coronaria y fibrilación auricular entre otras (48), teniendo en cuenta las características individuales de cada paciente y las recomendaciones de las guías de acuerdo a cada enfermedad.

El estudio TOPCAT (49), que evaluó el uso de la Espironolactona en pacientes con FEVI >45\%, no mostró un efecto benéfico en mortalidad y hospitalización para estos pacientes en los resultados generales. Sin embargo, el análisis de subgrupos encontró una disminución significativa en la hospitalización debido a falla cardiaca en pacientes provenientes de las Américas que habían sido admitidos solo por elevación de los péptidos natriureticos (BNP y NTproBNP) (50). Se requieren más estudios para generalizar su uso, dado que la eficacia potencial de la Espironolactona se asoció a una discrepancia en las tasas de eventos con placebo y el beneficio fue mayor en pacientes con fracciones de eyección del VI <50\% (49,51).

Con respecto al uso de beta bloqueadores se ha identificado disminución en mortalidad de origen cardiovascular, principalmente en estudios observacionales $(51,53)$, pero se considera que el efecto benéfico del fármaco se debe a una reducción del $25 \%$ en la enfermedad cardiovascular y no es posible generalizar este resultado global, dada la reducida población de pacientes con FEVI mayor al $40 \%$ en los estudios. Por otra parte, una revisión Cochrane del 2018 considera que no hay diferencias significativas cuando se comparan estos fármacos con placebo. Respecto a los inhibidores del eje renina-angiotensina-aldosterona no existen resultados concluyentes, por lo que no hay indicación de su uso diferente al control de comorbilidades $(51,52)$.

Uno de los fármacos de los cuales se esperaban buenos resultados, es el grupo de los inhibidores de la neprilisina (ARNI). Sin embargo, este no demostró reducción importante en desenlaces de hospitalización por IC, ni mortalidad cardiovascular, a diferencia de pacientes con ICFEr (54).

Debido a la falta de intervenciones con efectos significativos en mortalidad, en comparación con los resultados observados en ICFEr, se ha empezado a considerar la importancia del tratamiento con base en fenotipos determinados por medio de características fisiopatológicas predominantes en determinadas subpoblaciones. En 2016, Sanjiv et $a l$. propusieron un esquema de manejo que inicia con recomendaciones farmacológicas para el fenotipo con mayor importancia (congestión pulmonar / síndrome metabólico) 
Tabla 1. Propuesta de manejo de ICFEp según diferentes fenotipos

\begin{tabular}{|c|c|c|c|c|c|}
\hline \multirow{2}{*}{$\begin{array}{l}\text { Fenotipos de } \\
\text { predisposición }\end{array}$} & \multicolumn{5}{|c|}{ Fenotipos clínicos } \\
\hline & $\begin{array}{l}\text { Congestión } \\
\text { Pulmonar }\end{array}$ & $\begin{array}{l}\text { Incompetencia } \\
\text { Cronotrópica }\end{array}$ & $\begin{array}{l}\text { Hipertensión } \\
\text { Pulmonar }\end{array}$ & $\begin{array}{l}\text { Debilidad } \\
\text { Muscular }\end{array}$ & $\begin{array}{l}\text { Fibrilación } \\
\text { Auricular }\end{array}$ \\
\hline Síndrome metabólico & $\begin{array}{l}\text { Diuréticos* } \\
\text { Estatinas } \\
\text { Sacubitril } \\
\text { Espironolactona }\end{array}$ & $\begin{array}{l}\text { Marcapasos adap- } \\
\text { table }\end{array}$ & $\begin{array}{l}\text { Vasodilatador } \\
\text { pulmonar }\end{array}$ & $\begin{array}{l}\text { Programa de } \\
\text { Ejercicio }\end{array}$ & $\begin{array}{l}\text { Cardioversión } \\
\text { (CV) o Control de } \\
\text { frecuencia (CF) + } \\
\text { Anticoagulación }\end{array}$ \\
\hline Hipertensión arterial & IECAS o ARAS & $\begin{array}{l}\text { IECAS o ARAS + } \\
\text { Marcapasos adap- } \\
\text { table }\end{array}$ & IECAS o ARAS & $\begin{array}{l}\text { IECAS o ARAS } \\
+ \text { Programa de } \\
\text { Ejercicio }\end{array}$ & $\begin{array}{l}\text { IECAS o ARAS + } \\
\text { CV o CF + Anticoa- } \\
\text { gulación }\end{array}$ \\
\hline Disfunción renal & Soporte renal & $\begin{array}{l}\text { Soporte renal + } \\
\text { Marcapasos adap- } \\
\text { table }\end{array}$ & $\begin{array}{l}\text { Soporte renal + } \\
\text { Vasodilatador } \\
\text { pulmonar }\end{array}$ & $\begin{array}{l}\text { Soporte renal } \\
+ \text { Programa de } \\
\text { Ejercicio }\end{array}$ & $\begin{array}{l}\text { Soporte renal + } \\
\text { CV o CF + Anticoa- } \\
\text { gulación }\end{array}$ \\
\hline Enfermedad coronaria & $\begin{array}{l}\text { IECAS + } \\
\text { Revascularización }\end{array}$ & $\begin{array}{l}\text { Revascularización + } \\
\text { IECAS + Marcapasos } \\
\text { adaptable }\end{array}$ & $\begin{array}{l}\text { Revascularización } \\
+ \text { IECAS + } \\
\text { Vasodilatador } \\
\text { pulmonar }\end{array}$ & $\begin{array}{l}\text { Revascularización } \\
\text { + IECAS + Progra- } \\
\text { ma de Ejercicio }\end{array}$ & $\begin{array}{l}\text { IECAS + Revascu- } \\
\text { larización + CV o } \\
\text { CF + Anticoagu- } \\
\text { lación }\end{array}$ \\
\hline
\end{tabular}

*Diuréticos de asa Traducido y adaptado de referencia 46 (sin permiso).

por estar presente en más del $80 \%$ de los pacientes con falla cardiaca con ICFEp (Tabla 1). A partir de este punto, se sugieren manejos adicionales dependiendo de la presencia de otras características predisponentes (localizadas en la parte inferior de la Tabla 1) o de presentaciones clínicas adicionales (hacia la derecha de la misma) (51). Esta perspectiva abre una serie de nuevos objetivos terapéuticos que, a futuro, pueden impulsar avances en el tratamiento y la prevención de este síndrome.

Se listan los diferentes fenotipos clínicos y predisposición con el manejo recomendado para cada combinación. Se inicia con tratamiento para congestión pulmonar con síndrome metabólico y se adicionan manejos hacia la derecha y hacia abajo según sea el caso.

\section{CONCLUSIONES}

El síndrome de insuficiencia cardiaca con fracción de eyección preservada constituye un reto actual en la práctica clínica, dado que se genera de mecanismos fisiopatológicos distintos a los ya conocidos en pacientes con fracción de eyección reducida, produciendo dificultades en su abordaje y manejo. La mayoría de estudios dirigidos a esta población han mostrado efectos globales neutros en la morbilidad y mortalidad. En la actualidad, se sugiere que posterior al diagnóstico cada paciente sea manejado por fenotipos clínicos y enfocados en los factores de riesgo que propiciaron el desarrollo de este síndrome. Finalmente, se esperan nuevos estudios que logren identificar intervenciones terapéuticas con un impacto favorable.

\section{CONFLICTO DE INTERESES}

Ninguno de los autores declara conflicto de intereses.

\section{FINANCIACIÓN}

Ninguna fuente de financiación.

\section{REFERENCIAS}

1. Redfield MM. Heart Failure with Preserved Ejection Fraction. The New England journal of medicine. 2016; 375(19): 1868-1877. https://doi.org/10.1056/ NEJMcp1511175

2. Zakeri R, Cowie MR. Heart failure with preserved ejection fraction: controversies, challenges and future directions. 
Heart. 2018; 104(5):377-384. https://doi.org/10.1136/ heartinl-2016-310790

3. Ponikowski P, Voors A, Anker S, Bueno H, Cleland J, Coats A et al. 2016 ESC Guidelines for the diagnosis and treatment of acute and chronic heart failure. European Journal of Heart Failure. 2016;18(8):891-975. https://doi. org/10.1002/ejhf.592

4. Oktay AA, Shah SJ. Diagnosis and management of heart failure with preserved ejection fraction: 10 key lessons. Current cardiology reviews, 2015; 11(1): 42-52. https://doi.org/10.2174 /1573403X09666131117131217

5. Oktay AA, Rich JD, Shah SJ. The emerging epidemic of heart failure with preserved ejection fraction. Current heart failure reports. 2013; 10(4): 401-410. https://doi. org/10.1007/s11897-013-0155-7

6. Gazewood JD, Turner PL. Heart Failure with Preserved Ejection Fraction: Diagnosis and Management. American family physician. 2017; 96(9): 582-588.

7. Dunlay SM, Roger VL, Redfield MM. Epidemiology of heart failure with preserved ejection fraction. Nature Reviews Cardiology, 2017, 14(10), 591-602. https://doi. org/10.1038/nrcardio.2017.65

8. Gómez E. Identificación de pacientes con falla cardiaca y función sistólica preservada: Un estudio epidemiológico regional (I-PREFER Study) - Colombia. Revista Colombiana de Cardiología. 2013;20(2):58-67. https:// doi.org/10.1016/S0120-5633(13)70027-5

9. Gladden JD, Linke WA, Redfield MM. Heart failure with preserved ejection fraction. Pflugers Archiv: European journal of physiology. 2014; 466(6): 1037-1053. https:// doi.org/10.1007/s00424-014-1480-8

10. Oren 0, Goldberg S. Heart Failure with Preserved Ejection Fraction: Diagnosis and Management. The American Journal of Medicine. 2017; 130(5): 510-516. https://doi. org/10.1016/j.amjmed.2016.12.031

11. Pfeffer MA, Shah AM, Borlaug BA. Heart Failure with Preserved Ejection Fraction in Perspective. Circulation research. 2019; 124(11): 1598-1617. https://doi. org/10.1161/CIRCRESAHA.119.313572

12. Gerber Y, Weston SA, Redfield MM, Chamberlain AM, Manemann SM, Jiang R, Killian JM, Roger VL. A contemporary appraisal of the heart failure epidemic in Olmsted County, Minnesota, 2000 to 2010. JAMA internal medicine. 2015; 175(6): 996-1004. https://doi. org/10.1001/jamainternmed.2015.0924

13. Vaduganathan M, Patel RB, Michel A, Shah SJ, Senni M., Gheorghiade M, Butler J. Mode of Death in Heart Failure with Preserved Ejection Fraction. Journal of the American College of Cardiology. 2017; 69(5): 556-569. https://doi.org/10.1016/j.jacc.2016.10.078
14. Tibrewala A, Yancy CW. Heart Failure with Preserved Ejection Fraction in Women. Heart Failure Clinics. 2019; 15(1): 9-18. https://doi.org/10.1016/j.hfc.2018.08.002

15. Gupta DK, Shah AM, et al. Heart failure with preserved ejection fraction in African Americans: The ARIC (Atherosclerosis Risk in Communities) study.JACC. Heart failure. 2013; 1(2): 156-163. https://doi.org/10.1016/j. jchf.2013.01.003

16. Lai YC, Wang L, Gladwin MT. Insights into the pulmonary vascular complications of heart failure with preserved ejection fraction. The Journal of physiology. 2019; 597(4): 1143-1156. https://doi.org/10.1113/JP275858

17. Borlaug BA. The pathophysiology of heart failure with preserved ejection fraction. Nature reviews. Cardiology. 2014; 11(9): 507-515. https://doi.org/10.1038/ nrcardio.2014.83

18. Cikes M, Claggett B, Shah AM. et al. Atrial Fibrillation in Heart Failure With Preserved Ejection Fraction: The TOPCAT Trial. JACC. Heart failure. 2018; 6(8): 689-697. https://doi.org/10.1016/j.jchf.2018.05.005

19. Tromp J, MacDonald MR, et al. Heart Failure with Preserved Ejection Fraction in the Young. Circulation. 2018; 138(24): 2763-2773. https://doi.org/10.1161/ CIRCULATIONAHA.118.034720

20. Paulus WJ, Tschöpe C. A novel paradigm for heart failure with preserved ejection fraction: comorbidities drive myocardial dysfunction and remodeling through coronary microvascular endothelial inflammation. Journal of the American College of Cardiology. 2013; 62(4): 263-271. https://doi.org/10.1016/j. jacc.2013.02.092

21. Obokata M, Olson TP, Reddy Y, Melenovsky V, Kane GC, Borlaug B. A. Haemodynamics, dyspnoea, and pulmonary reserve in heart failure with preserved ejection fraction. European heart journal. 2018; 39(30): 2810-2821. https://doi.org/10.1093/eurheartj/ehy268

22. Zile MR, Litwin S. Heart Failure with a Preserved Ejection Fraction. Robert B., Mann D., Tomaselli G. Braunwald's Heart Disease: A Textbook of Cardiovascular Medicine. 11 ed. ELSEVIER. Philadelphia, PA. 2019; P. 523-542.

23. Reddy Y, Andersen MJ, Obokata M, Koepp K. E, Kane GC, Melenovsky V, Olson TP, Borlaug BA. Arterial Stiffening with Exercise in Patients with Heart Failure and Preserved Ejection Fraction. Journal of the American College of Cardiology. 2017; 70(2): 136-148. https://doi. org/10.1016/j.jacc.2017.05.029

24. Lam C, Voors AA, de Boer RA, Solomon SD, van Veldhuisen DJ. Heart failure with preserved ejection fraction: from mechanisms to therapies. European heart journal. 2018; 39(30): 2780-2792. https://doi. org/10.1093/eurheartj/ehy301 
25. Borlaug BA, Olson TP, Lam CS, Flood KS, Lerman A, Johnson BD, Redfield MM. Global cardiovascular reserve dysfunction in heart failure with preserved ejection fraction. Journal of the American College of Cardiology. 2010; 56(11): 845-854. https://doi.org/10.1016/j. jacc.2010.03.077

26. Obokata M, Reddy Y, Pislaru SV, Melenovsky V, Borlaug BA. Evidence Supporting the Existence of a Distinct Obese Phenotype of Heart Failure With Preserved Ejection Fraction. Circulation. 2017; 136(1): 6-19. https:// doi.org/10.1161/CIRCULATIONAHA.116.026807

27. Plitt GD, Spring JT, Moulton MJ, Agrawal DK. Mechanisms, diagnosis, and treatment of heart failure with preserved ejection fraction and diastolic dysfunction. Expert review of cardiovascular therapy. 2018; 16(8): 579-589. https:// doi.org/10.1080/14779072.2018.1497485

28. Lekavich CL, Barksdale DJ, Neelon V, Wu JR. Heart failure preserved ejection fraction (HFpEF): an integrated and strategic review. Heart Failure Reviews. 2015; 20(6): 643653. https://doi.org/10.1007/s10741-015-9506-7

29. Gladden JD, Chaanine AH, Redfield MM. Heart Failure with Preserved Ejection Fraction. Annual Review of Medicine. 2018; 69(1): 65-79. https://doi.org/10.1146/ annurev-med-041316-090654

30. Upadhya B, Kitzman DW. Heart failure with preserved ejection fraction: New approaches to diagnosis and management. Clinical cardiology. 2020; 43(2): 145-155. https://doi.org/10.1002/clc.23321

31. Shah AM, et al. PARAGON-HF Investigators. Echocardiographic Features of Patients with Heart Failure and Preserved Left Ventricular Ejection Fraction. Journal of the American College of Cardiology. 2019; 74(23): 2858-2873. https://doi.org/10.1016/j.jacc.2019.09.063

32. Huis In 't Veld AE, de Man FS, van Rossum AC, Handoko ML, Handoko ML. How to diagnose heart failure with preserved ejection fraction: the value of invasive stress testing. Neth Heart J. 2016; 24(4): 244-51. https://doi.org/10.1007/ s12471-016-0811-0

33 Meijers WC, van der Velde AR, de Boer RA. Biomarkers in heart failure with preserved ejection fraction. Netherlands heart journal: monthly journal of the Netherlands Society of Cardiology and the Netherlands Heart Foundation. 2016; 24(4): 252-258. https://doi. org/10.1007/s12471-016-0817-7

34. Tanase DM, Radu S, Al Shurbaji S, Baroi GL, FloridaCostea C, Turliuc MD, Ouatu A, Floria M. Natriuretic Peptides in Heart Failure with Preserved Left Ventricular Ejection Fraction: From Molecular Evidences to Clinical Implications. International journal of molecular sciences. 2019; 20(11): 2629. https://doi.org/10.3390/ ijms20112629
35. Anjan VY, Loftus TM, Burke MA, Akhter N, Fonarow GC, Gheorghiade M, Shah SJ. Prevalence, clinical phenotype, and outcomes associated with normal B-type natriuretic peptide levels in heart failure with preserved ejection fraction. The American journal of cardiology. 2012; 110(6): 870-876. https://doi.org/10.1016/j. amjcard.2012.05.014

36. Buckley LF, Canada JM, et al. Low NT-proBNP levels in overweight and obese patients do not rule out a diagnosis of heart failure with preserved ejection fraction. ESC heart failure. 2018; 5(2): 372-378. https:// doi.org/10.1002/ehf2.12235

37. Meijers WC, Hoekstra T, Jaarsma T, van Veldhuisen DJ, de Boer RA. Patients with heart failure with preserved ejection fraction and low levels of natriuretic peptides. Neth Heart J. 2016; 24(4): 287-95. https://doi.org/10.1007/ s12471-016-0816-8

38. Reddy Y, Carter RE, Obokata M, Redfield MM, Borlaug BA. A Simple, Evidence-Based Approach to Help Guide Diagnosis of Heart Failure with Preserved Ejection Fraction. Circulation. 2018; 138(9): 861-870. https:// doi.org/10.1161/CIRCULATIONAHA.118.034646

39. Sueta D, Yamamoto E, Nishihara T, et al. H2FPEF Score as a Prognostic Value in HFpEF Patients. American journal of hypertension. 2019; 32(11): 1082-1090. https://doi. org/10.1093/ajh/hpz108

40. Burkert Pieske, Carsten Tschöpe, et al. How to diagnose heart failure with preserved ejection fraction: the HFAPEFF diagnostic algorithm: a consensus recommendation from the Heart Failure Association (HFA) of the European Society of Cardiology (ESC), European Heart Journal. 2019; 40(40): 3297-3317, https://doi.org/10.1093/ eurheartj/ehz641

41. Barandiarán-Aizpurua A, Sanders-van Wijk S, et al. Validation of the HFA-PEFF score for the diagnosis of heart failure with preserved ejection fraction. European journal of heart failure. 2020; 22(3): 413-421. https:// doi.org/10.1002/ejhf.1614

42. Iwano H, Little W. Heart failure: What does ejection fraction have to do with it? Journal of Cardiology. 2013; 62(1):1-3. https://doi.org/10.1016/j.jjcc.2013.02.017

43. Mentz RJ, Kelly JP, et al. Noncardiac comorbidities in heart failure with reduced versus preserved ejection fraction. Journal of the American College of Cardiology. 2014; 64(21): 2281-2293. https://doi.org/10.1016/j. jacc.2014.08.036

44. Hegde S, Claggett B, Shah A, Lewis E, Anand I, Shah S, et al. Physical Activity and Prognosis in the TOPCAT Trial. Circulation. 2017; 136(11): 982-992. https://doi. org/10.1161/CIRCULATIONAHA.117.028002 
45. Pandey A, Parashar A, Kumbhani D, Agarwal S, Garg J, Kitzman D, Levine B, Drazner M, Berry J. Exercise training in patients with heart failure and preserved ejection fraction: meta-analysis of randomized control trials. Circulation. Heart failure. 2015; 8(1): 33-40. https://doi.org/10.1161/ CIRCHEARTFAILURE.114.001615

46. Shah SJ, Kitzman DW, Borlaug BA, van Heerebeek L, Zile MR, Kass DA, Paulus WJ. Phenotype-Specific Treatment of Heart Failure with Preserved Ejection Fraction: A Multiorgan Roadmap. Circulation. 2016; 134(1): 73-90. https://doi.org/10.1161/CIRCULATIONAHA.116.021884

47. Pandey A, Patel KV, Vaduganathan M, Sarma S, Haykowsky MJ, Berry JD, Lavie CJ. Physical Activity, Fitness, and Obesity in Heart Failure with Preserved Ejection Fraction. JACC. Heart failure. 2018; 6(12): 975982. https://doi.org/10.1016/j.jchf.2018.09.006

48. Yancy C, Jessup M, Bozkurt B, Butler J, Casey D, Drazner M, et al, ACCF/AHA Guideline for the Management of Heart Failure. Circulation. 2013; 128(16): 240-327 https://doi.org/10.1161/CIR.0b013e31829e8776

49. Pitt B, Pfeffer MA, Assmann SF, et al. Spironolactone for heart failure with preserved ejection fraction. $\mathrm{N}$ Engl J Med. 2014; 370(15):1383-1392. https://doi.org/10.1056/ NEJMoa1313731

50. de Denus S, O'Meara E, et al. Spironolactone Metabolites in TOPCAT - New Insights into Regional Variation. The New England journal of medicine. 2017; 376(17): 16901692. https://doi.org/10.1056/NEJMc1612601

51. Zheng SL, Chan FT, Nabeebaccus AA, Shah AM, McDonagh T, Okonko D. O, Ayis S. Drug treatment effects on outcomes in heart failure with preserved ejection fraction: a systematic review and meta-analysis. Heart (British Cardiac Society). 2018; 104(5): 407-415. https:// doi.org/10.1136/heartjnl-2017-311652

52. Martin N, Manoharan K, Thomas J, Davies C, Lumbers RT. Beta-blockers and inhibitors of the reninangiotensin aldosterone system for chronic heart failure with preserved ejection fraction. The Cochrane database of systematic reviews. 2018; 6(6): CD012721. https://doi.org/10.1002/14651858.CD012721.pub2

53. Bavishi C, Chatterjee S, Ather S, Patel D, Messerli FH. Beta-blockers in heart failure with preserved ejection fraction: a meta-analysis. Heart failure reviews. 2015; 20(2): 193-201. https://doi.org/10.1007/s10741-0149453-8

54. Solomon SD, McMurray J, et al. PARAGON-HF Investigators and Committees. Angiotensin-Neprilysin Inhibition in Heart Failure with Preserved Ejection Fraction. The New England journal of medicine. 2019; 381(17): 1609-1620. https://doi.org/10.1056/NEJMoa1908655 